\title{
Single-Cell Transcriptome Analysis of Developing and Regenerating Spiral Ganglion Neurons
}

\author{
Kelvin Y. Kwan ${ }^{1,2}$
}

Published online: 4 August 2016

(C) Springer International Publishing AG 2016

\begin{abstract}
The spiral ganglion neurons (SGNs) of the cochlea are essential for our ability to hear. SGN loss after exposure to ototoxic drugs or loud noise results in hearing loss. Pluripotent stem cell-derived and endogenous progenitor cell types have the potential to become SGNs and are cellular foundations for replacement therapies. Repurposing transcriptional regulatory networks to promote SGN differentiation from progenitor cells is a strategy for regeneration. Advances in the Fludigm C1 workflow or Drop-seq allow sequencing of single-cell transcriptomes to reveal variability between cells. During differentiation, the individual transcriptomes obtained from single-cell RNA-seq can be exploited to identify different cellular states. Pseudotemporal ordering of transcriptomes describes the differentiation trajectory, allows monitoring of transcriptional changes, and determines molecular barriers that prevent the progression of progenitors into SGNs. Analysis of single-cell transcriptomes will help develop novel strategies for guiding efficient SGN regeneration.
\end{abstract}

Keywords Spiral ganglion neurons $\cdot$ Regeneration $\cdot$ Inner ear development $\cdot$ Cochlea $\cdot$ Single-cell RNA-seq

This article is part of the Topical Collection on Bioinformatics and Stem Cell

Kelvin Y. Kwan

Kwan@dls.rutgers.edu

1 Department of Cell Biology and Neuroscience, Rutgers University, 604 Allison Rd., Nelson Labs D250, Piscataway, NJ 08854, USA

2 Stem Cell Research Center and Keck Center for Collaborative Neuroscience, Rutgers University, Piscataway, NJ 08854, USA

\section{Introduction}

The organs of the inner ear are responsible for our ability to hear and balance. Encased in a bony labyrinth, the cochlea is responsible for our ability to discriminate and hear complex sounds. Residing within the cochlear sensory epithelia are a single row of inner hair cells (IHCs) and three rows of outer hair cells (OHCs). The IHCs transform sounds into neural signals while OHCs amplify sound [1-3]. The spiral ganglion neurons (SGNs) are the primary auditory neurons. Type I SGNs comprise $95 \%$ of the SGN population that extend single, unbranched, myelinated neurites to innervate a single IHC while the remaining $5 \%$ of SGNs are type II neurons that possess thin, unmyelinated fibers that innervate OHCs en passant [4-8]. The IHCs are responsible for encoding sound stimuli and are exclusively innervated by multiple type I SGNs [8]. Release of neurotransmitters from IHCs onto peripheral endings of type I SGNs initiates transmission of neural signal to the auditory circuit $[9,10]$. The mature human cochlea contains only 16,000 hair cells and 30,000 SGNs. Built for exquisite sensitivity and speed, hair cells and SGNs have a high metabolic demand and possess delicate cellular structures that make them prime targets for ototoxic damage. Insults that cause hair cell death, such as loud noises, aminoglycosides, or chemotherapeutic agents, also cause acute loss of specialized synapses between IHCs and SGNs, retraction of the nerve terminal, followed by delayed degeneration of SGN over months or years [11-13].

Hearing loss affects $13 \%$ or $\sim 30$ million people in the USA who are 12 years of age or older [14]. Given a population who are exposed to loud sounds from portable music players and treated with ototoxic drugs for cancer therapies or infections, hearing loss due to cochlear hair cell or SGN degeneration will increase as a major health concern. In addition to the environmental effects on hearing loss, genetic mutations resulting in 
hearing loss have been invaluable in identifying genes involved in hair cell or SGN development, function, and maintenance $[15,16]$. One of the leading challenges in alleviating hearing loss is cellular replacement of SGNs. Successful auditory prostheses such as hearing aids and cochlear implants bypass hair cell function but depend on existing SGNs to initiate the auditory signal. Efforts in repurposing transcriptional regulatory networks and signaling pathways for cellular regeneration have been employed despite potential differences between the function of these pathways during development and regeneration [17].

\section{Development of SGNs}

During embryonic development, the mammalian inner ear undergoes dramatic morphological and cellular changes. The mouse inner ear starts from the thickening of the ectodermal layer of cells between rhombomeres 5 and 6 to form the otic placode at embryonic day (E)9.5. As development proceeds, the otic placode invaginates to form an otic cup and closes at E10.5 to form the otic vesicle [18]. The otic vesicle undergoes an elaborate morphogenesis that is intimately coupled with specification of the sensory cells and neurons that reside within the inner ear $[19,20]$. At $\sim$ E9.5, a subset of neurosensory precursors marked by Sox 2 and Neurog 1 delaminate from the antero-ventral portion of the otic cup to form the future cochlear vestibular ganglion (CVG). Little is known about the separation of the CVG into the spiral ganglion and the vestibular ganglion; however, cells that give rise to the two ganglia are likely distinct by E10.5 since neurites extending from the two ganglia are clearly distinguishable by E11.5-12.5 [21]. In the developing cochlea, neuroblasts delaminate throughout the growing cochlear duct starting from the future ductus reuniens region, then the middle region and finally from the apex of the cochlear duct $[22,23]$. Delaminating neuroblasts continue dividing within the nascent cochlea ganglion. Cell cycle exit initiates from the base of the cochlea at $\sim$ E10.5 and proceeds in a wave towards the apex at $\sim \mathrm{E} 12.5$. By E14.5, all the neuroblasts are post-mitotic [24, 25].

The cochlear ganglion gives rise to the SGNs and by E12.5, the neurons start extending neurites towards hair cells. The early neuronal differentiation process involves the Sox2-Neurog1NeuroD1 transcriptional network [26, 27]. Mutations in human Sox2 result in anophthalmia, a severe eye malformation, associated with sensorineural hearing loss in patients [28, 29]. Similarly, hypomorphic Sox2 mice, yellow submarine (Ysb) and light coat circler (Lcc), lack Sox 2 expression in the inner ear and are devoid of hair cells, supporting cells and SGNs [30, 31]. Animals lacking Neurog1 are devoid of afferent and efferent fibers but retain morphologically normal hair cells [32]. In NeuroD1 null animals, a defect in differentiation and survival results in the massive loss of SGNs [33]. In addition to their individual roles during SGN development, feedback inhibition of Sox 2 expression by Neurog1 and NeuroD1 is required for proper progression of otic neurogenesis [26]. For an in depth review of early stages of cochlea and vestibular neuron development, the readers are referred to several reviews [18, 34, 35].

After SGNs are formed, the general wiring pattern of neurites is established at $\sim$ E15.5 [36]. The neurites innervate the basolateral surface of the hair cells, seek synaptic partners, and establish functional contacts. SGNs have to encode the frequency, intensity, and timing of neural transmitter release from hair cells and communicate this information rapidly and faithfully to the brain [34]. To ensure rapid and precise neurotransmission, SGNs form a specialized ribbon synapse structure with IHCs [37-39]. Ribbon synapses contain an electron-dense multi-protein ribbon structure, which tethers a large pool of readily releasable vesicles for fast and sustained release of glutamate from the IHCs [40-42]. SGNs respond to fast glutamate release due to abundant clusters of AMPA-type receptors at the post-synaptic density (PSD) which allows for matched responses [43]. During the late stages of SGN development, the Gata3-MafB transcriptional regulatory network serves to coordinate SGN differentiation with ribbon synapse formation [44]. Haploinsufficiency of Gata3 causes hypoparathyroidism, deafness, and renal dysplasia (HDR) syndrome humans [45]. At E13.5, Gata3 can be detected in the cochlea, which includes the developing SGNs [46, 47]. Ablation of Gata3 at different time points during SGN development affects formation of neurosensory cells, causes aberrant neurite outgrowth, and decreases SGN survival [48-51]. Gata3 is likely involved in both specifying and maintaining SGN cell fate as well as establishing the auditory circuit $[48,51]$. Loss of Gata3 affects expression of many genes including Mafb [48]. Mafb is part of a transcription factor family implicated in terminal differentiation [52]. Deletion of MafB early during SGN development using a Neurog 1 Cre animal does not affect the initial production of SGNs but is crucial for later stages of differentiation [44].

Synaptogenesis between IHCs and SGNs occurs at E17.5, late in SGN differentiation [53]. Components of pre-synaptic ribbons proteins are initially dispersed throughout the IHC cytoplasm and eventually coalesce at the basolateral surface, opposite to the SGN post-synaptic terminals [5]. In parallel, SGN terminals mature to form large post-synaptic densities containing large numbers of the AMPA receptors GluR2 and GluR3 $[6,43]$. The number of synapses between hair cells and SGNs peaks at post-natal day (P)6 and is followed by refinement. At the onset of hearing at P12, most ribbons are juxtaposed to a single SGN terminal [5]. Concurrent with synaptogenesis, SGNs fire action potentials as early as E14 and become electrophysiologically mature after birth [53]. The mature SGNs form at least two different populations of neurons that differ in their spontaneous discharge rate and threshold to acoustic stimulation [54]. These are the low spontaneous 
discharging high threshold fibers and the high spontaneous discharging low threshold fibers [54]. The mature SGNs form the auditory nerve fiber that conveys neural signal from the inner hair cells to targets in the cochlear nucleus. Loss of MafB in SGNs results in the loss of well-defined post-synaptic structures and shows a decrease in GluR2 expression at the afferent endings [44]. These results implicate MafB in forming post-synaptic densities at the ribbon synapse between IHCs and SGNs. Overexpression of MafB conversely accelerates maturation of the SGNs by allowing AMPA receptors to cluster earlier as observed by increased expression of GluR2 at the afferent terminals [44].

The Sox2-Neurog1-NeuroD1 transcriptional regulatory network is involved in specification, neurite extension, and survival of SGNs while the Gata3-MafB is involved in specification, circuit formation, and synaptogenesis. The handful of transcription factors provides a rudimentary transcriptional regulatory network for SGN development and potential factors that drive SGN differentiation. Repurposing these transcription factors to generate SGNs from different progenitor cells has been employed as a strategy to promote regeneration.

\section{Otic Progenitors for Cell Replacement and Direct Conversion of SGNs}

Many different stem cell or progenitor cell types obtained from the inner ear show competency to become neurons and are potential cellular sources for replacing SGNs [55-57]. Embryonic stem cell-derived inner ear organoids can develop otic vesicles containing hair cells that are innervated by neurons through modulation of Fgf, Bmp, and Wnt signaling pathways using small molecule inhibitors and ligands [58]. Cells derived from different portions of the sensory organs form otospheres when cultured in vitro and show limited self-renewal properties but are competent to differentiate into neurons and hair cells $[55,57,59]$. Non-sensory cells that reside within the cochlear epithelium are another source of neuronal competent cells [30,60].

To determine if exogenous stem cells can be used to replace SGNs, ouabain, a plant-derived arrow toxin, has been used to specifically ablate type I SGNs from cochlear explants in vitro $[61,62]$. Oubain-induced SGN loss has been employed to establish a mouse model of neuropathy and was shown to only cause death of type I SGNs [63]. Introduction of ES-derived progenitors into the nerve trunk of animals after oubaininduced neuropathy allows for cell engraftment, neuronal differentiation, and extension of neurites peripherally to hair cells and centrally to the cochlear nucleus $[64,65]$. These studies show that stem cell-derived progenitors have the capability to differentiate into neurons even when residing in a damaged niche. Auditory brain stem recordings (ABR) of animals after receiving progenitor cell replacement therapy show that some animals display partial recovery of auditory responses [65]. Understanding the molecular transitions of progenitor cells into SGNs will be invaluable for accelerating regenerative efforts.

Previous studies have used otic progenitors to demonstrate the ability to re-innervate hair cells and cells of the cochlear nucleus to re-form the auditory circuit [59, 64, 66, 67]. Although the progenitor state can be well defined, the transitional states and barriers towards achieving functional SGNs fate are much more ambiguous. One defined otic progenitor transcriptome is the immortalized multipotent otic progenitor cells (iMOP). iMOP cells are derived from embryonic cochlea, can self-renew, and are derived from single cells. iMOP cells can differentiate into functional hair cells and iMOP-derived neurons if given the appropriate cues [68]. To observe differentiation of progenitors in a damaged cochlea, co-cultures of iMOP cells with ouabain-denervated cochlea explants are used. Addition of ouabain to the murine cochlear explants ablates SGNs as shown by the lack of neuronal $\beta$ tubulin (Tubb3) labeled radial fibers that extend out from the modiolus (Fig. 1a, b). iMOP cells placed in the modiolar region can differentiate into bipolar iMOP-derived neurons. To mark engrafted iMOP cells in the damaged cochlear explants, a stable iMOP cell line expressing mCherry was generated. The mCherry expressing iMOP stable cell line allows tracking and visualizing the morphological features of live cells. iMOP-derived neurons possess two distinct neuronal processes that are distinguishable by the width of the neurites. In iMOP-derived neurons, mCherry fluorescence reveals a thin axon or the peripheral axon (P) that extends towards hair cells, while a thick and more intensely labeled central axon (C) projects away from hair cells (Fig. 1c). The twofold difference in width between the thinner peripheral axons and the central axons in SGNs has been observed across multiple animal species in vivo [69-71]. In iMOP-denervated cochlear explant co-cultures, Myo7a labels hair cell bodies while phalloidin highlights hair bundles (Fig. 1d) [72]. Upon close inspection of the synaptic region between IHCs and the iMOP-derived neurite, the peripheral ending terminates at the basal portion of the inner hair cell body (Fig. 1f). Of the iMOP-derived neurons that engraft in the modiolar region, 10\% (11 of 113) extend peripheral neurites reach the inner hair cells. In addition to proper axon guidance, the iMOP-derived neurons express post-synaptic markers. In untreated cochlea cultures, multiple Psd95 puncta corresponding to the post-synaptic complex of endogenous SGN can be identified surrounding the Myo7a-labeled inner hair cell bodies (Fig. 1g). Ouabain treatment eliminates Psd95 puncta by destroying the synapses between the inner hair cells and SGNs (Fig. 1h) as previously reported $[63,67]$. In iMOP-derived neurons and deafferented cochlear co-cultures, 20\% (21/113) iMOP-derived neurons re-innervate the inner hair cells and display Psd95 puncta at the base of the hair cell bodies (Fig. 1i). These results suggest 


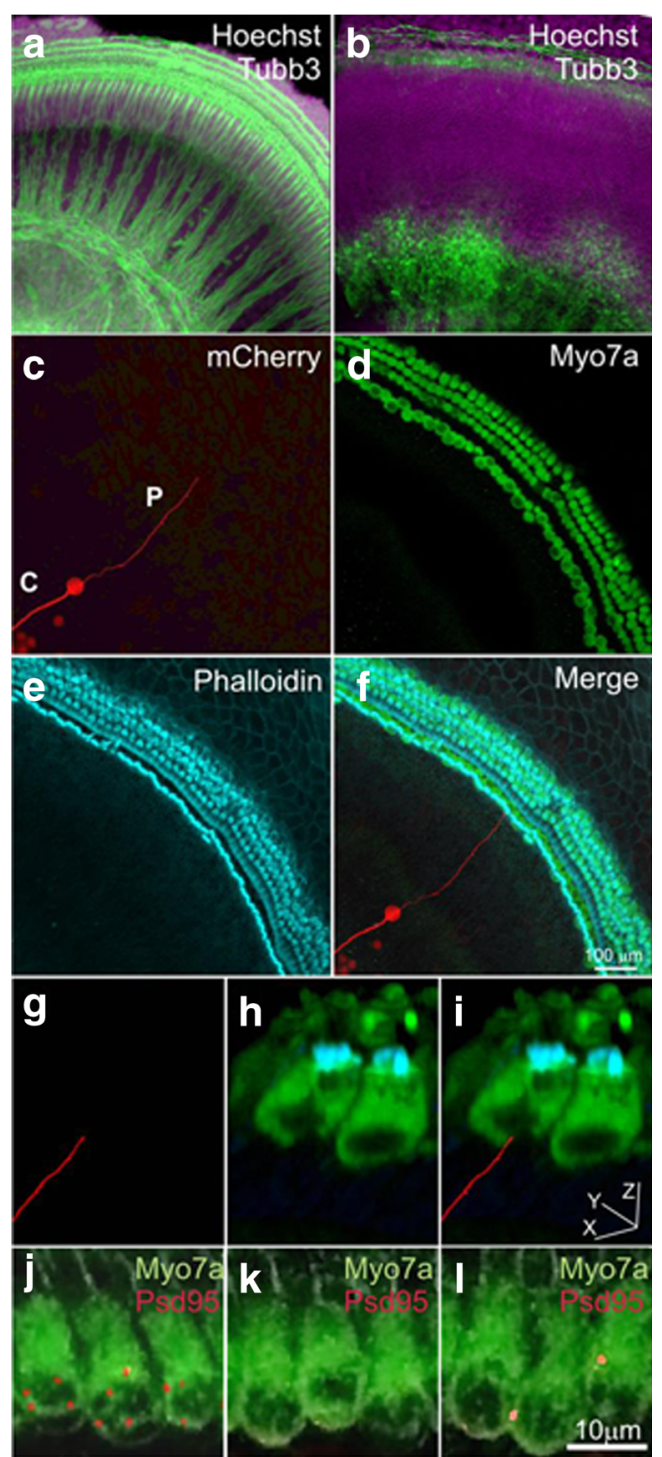

Fig. 1 iMOP-derived neurons and denervated cochlear co-cultures. a Hoechst-labeled nuclei (magenta) and Tubb3-labeled neurites from SGNs (green) in untreated cochlear explant. b Ouabain-treated cochlea lacking Tubb3-labeled SGN neurites. $\mathbf{c}$ iMOP-derived neuron expressing mCherry (red) with peripheral $(P)$ and central $(C)$ axons along with $\mathbf{d}$ Myo7a-labeled hair cells (green) with e phalloidin-marked hair bundles (cyan). $\mathbf{f}$ The merged image shows that the neuronal process terminates near inner hair cells. Magnified micrograph of $\mathbf{g}$ a mCherry-labeled neurite near the $\mathbf{h}$ basolateral portion of a Myo7a- and phalloidinlabeled hair cell. i Merged image suggests that the neurite terminates at the base of the hair cell body. Psd95 immunofluorescence (red) at the synaptic region between SGNs and hair cells (green) from $\mathbf{j}$ untreated cochlear explant, $\mathbf{k}$ ouabain-treated cochlear explant and $\mathbf{l}$ co-culture of oubain-denervated cochlear explant containing iMOP-derived neurons

that both cell autonomous and a permissive cellular environment contribute to neurite extension, guidance, and synapse formation. The co-culture preparation reveals a population of iMOP-derived neurons that successfully re-innervate hair cells as well as cells that fail to do so. Transcriptome comparison of these two cell populations will provide valuable information about the cell autonomous barriers that limit cellular processes essential for SGN replacement therapies.

Directed differentiation of cells in situ has been another avenue for regeneration. Non-sensory cells located within early post-natal cochlea retain the competency to be converted into neurons that fire action potentials, and express neuronal and synaptic proteins [60]. Overexpression of Ascll or a combination of Ascl1 with NeuroD1 allows non-sensory cochlear cells to become functional neurons [60]. Ascl1 is a pioneering factor that recruits other transcription factors to promote differentiation [73, 74]. Recruitment of endogenous transcription factors to promote otic neuronal differentiation may be one potential mechanism of converting non-sensory cells into SGNs. Overexpression of Sox 2 can also convert cochlear non-sensory cells into functional neurons with morphological and electrophysiological features similar to SGNs [30]. Overexpression of Sox2 may activate the Sox2-Neurog1NeuroD1 transcriptional regulatory network to drive SGN differentiation.

Molecular decisions made by engrafted or converted cells have important implications for regeneration. The competency of a progenitor cell determines the efficiency of conversion into a differentiated SGN. Improper molecular choices such as continued proliferation of stem cell-derived progenitors results in teratoma formation in the inner ear [75]. Improper differentiation may exacerbate an already compromised sensory system. One method for determining the events that occur during differentiation of progenitor cells into SGNs is by transcriptome profiling of single cells. Defining the competency and molecular determinants permissive for SGN differentiation from distinct progenitor cell types for SGN replacement therapies will be the next challenge.

\section{Establishing Cellular States of Inner Ear Cell Types by Transcriptome Profiling}

Transcriptome analysis of cells at different stages of SGN differentiation provides information about the molecular processes involved. During differentiation, RNA-seq of cells from both the developing embryo and stem cell-derived cultures offers a survey of the transcriptome. The diversity of developing otic cells or nuanced differences between closely related cell types cannot be determined by bulk RNA-seq. Bulk RNA-seq from a population of stem cells in culture provides information of the overall transcriptome state; however, the molecular heterogeneity of differentiating cells may be masked. Valuable information can be gleaned from the transcriptome of individual cells. The paucity of cells obtained from FACS sorted genetically labeled otic cells could potentially generate a pure population of cells but limited cell numbers affect the read-depth from bulk RNA-seq. Pluripotent derived inner ear organoids can recapitulate development of 
otic vesicles and generate hair cells and neurons but contain different cell types [58, 76]. Stem cell cultures allow generation of large numbers of cells for RNA-seq, but the heterogeneity within the cultures make transcriptome analysis from these cultures less informative. The number of iMOP cells that re-innervate hair cells from an in vitro regeneration paradigm are also very limited. The scarcity and heterogeneity of cells from these studies are less amenable to RNA-seq but particularly suitable for single-cell sequencing. Recent advances in single-cell RNA sequencing (scRNA-seq) such as Fluidigm C1 single-cell sequencing workflow or the Drop-seq allow parallel transcriptome sequencing of cells [77-82]. Transcriptome sequencing provides an unbiased method to define a cellular state which can be validated with immunolabeling or qPCR of previously established molecular markers. For reviews of scRNA-seq methods, workflow and data quality assessment, the readers are referred to recently published literature [83-86].

\section{Determining Transitional States by Pseudotemporal Ordering of Transcriptomes}

Development of a neurosensory precursor into a mature SGN may require transition through several intermediate states as defined by expression of molecular markers and residence in specific anatomical regions of the developing inner ear [18]. These cells become progressively fate restricted as they differentiate into SGNs. Achieving intermediate states will be milestones for productive differentiation of neurosensory precursors or pluripotent stem cells into SGNs. For direct conversion, cells directly transition from one cell fate to another [87]. Captured intermediate transcriptome states could reflect difficult transition points during the direct conversion process and provide information about molecular barriers that prevent efficient conversion into SGNs.

scRNA-seq or highly parallelized quantitative PCR (qPCR) methods can be employed to determine the different cellular states within a heterogeneous population of cells. Large-scale surveys of different cell types from scRNA-seq display the potential to reveal rare cell populations and lineage relationships. Cellular barcoding strategies allow increased parallelized sequencing of single cells at ultra-low depths [77]. Although the limitations of shallow sequencing may hinder identification of different cellular states, analysis of single-cell transcriptomes across shallow sequencing depths ( 50,000 reads per cell) allowed for unbiased cell-type classification of diverse cell types that included multiple progenitor and neuronal subtypes as well as biomarker identification in the developing cortex [88]. Using Drop-seq, sequencing of 44,808 single cells from the retina revealed at least 39 distinct cell populations that correlated well to the reported percentage cell types in the retina [56]. However, morphological and functional criteria estimate the presence of $\sim 100$ retinal cell types [89, 90]. Supervised analysis may help define the transcriptomes of closely associated or additional cell types. Increasing the sequencing depth and the number of cell sequenced may assist in saturated detection of distinct cell types in the retina.

Not only can scRNA-seq can provide a static depiction of different transcriptomes but it can also be used to glean temporal information within a differentiating culture. Defining different cellular transcriptomes in a population of differentiating cells permits prediction of a differentiation trajectory by ordering putative transitional transcriptome states. The idea to order static differential expression data to provide temporal information was first accomplished using microarray data [91]. From scRNA-seq data, differential gene expression data from genes that are either up or down regulated during differentiation can be defined. Software toolkits such as Monocle can use differentially expressed genes as temporal markers and place the transcriptomes in a pseudotemporal order to reflect the differentiation trajectory [92]. Using myoblast differentiation, temporal ordering of differentiating myoblasts has been accomplished by using an early marker for differentiation, myocyte enhancer factor 2c (Mef2c), and a late marker for differentiation myosin heavy chain (Myh2) to define the starting and ending cellular state [92]. Other advances in algorithms such as Sincell provides statistical assessment of the continuous hierarchy of intermediate cellular states obtained from scRNA-seq data in differentiating cells [93]. Many of the scRNA-seq software rely on known differential gene expression to guide placement of the transcriptomes in order to describe the progression of temporal events. More recent studies have used other bioinformatics pipelines such as Waterfall to determine how neural stem cells transition from quiescence to activation after brain injury or during adult neurogenesis [94, 95]. Unlike Monocle, Waterfall does not require prior information such as temporal delineators or well-defined markers to determine the starting and ending cellular states. For specialized transcripts that show oscillatory function such as cell cycle-dependent or circadian rhythm genes, Oscope provides a platform for identifying such genes from asynchronous cultures. Similar to the algorithms employing scRNA-seq to attain temporal data, Oscope exploits the presence of multiple cellular states in a heterogenous culture, applies a sinusoidal function to all gene pairs and identifies the best fit groups as putative oscillating genes [96]. Single-cell analysis can be extended from transcriptomes to proteomes. Wanderlust, a single-cell proteome algorithm uses single-cell mass spectroscopy data to generate a differentiation trajectory. Proteome analysis of human B cell undergoing lymphopoeiesis reveals a continuum of transitional states with discrete stages during $\mathrm{B}$ cell development [97].

Studies that apply scRNA-seq and parallelized single-cell qPCR analysis have just started for otic cells. Compared to 
other organs, such as the eye, the inner ear is small and difficult to access. The limited number of cells obtained from the inner ear has restricted transcriptome studies. Using more refined methodologies that employ genetic labeling of otic cells with fluorescent proteins allow visual identification, and permit selection of otic cells and exclusion of non-otic cells for scRNA-seq. To establish the different population of cells in the inner ear, cells from post-natal cochleae have been used [98]. A $\mathrm{Lfng}^{\mathrm{EGFP}}$; $\mathrm{Gfil}^{\mathrm{Cre}}{ }^{\mathrm{C}}$ R26R ${ }^{\text {CAG-tdTomato }}{ }^{\text {-triple transgenic mice }}$ [99-101] allow hair cells to express tdTomato and most nonsensory supporting cells to express EGFP. Microdissection, FACS sorting cells, and sequencing of 91 cochlear single cells provided a set of single-cell transcriptome data. Performing principal component analysis and clustering reveals four clusters of cells. The four groups include tdTomato cells that express Pou4f3, Pvalb, Bdnf, Otof, and Myo7a and EGFP cells that express Lfng, Wnt7a, Fgfr3, Proxl, and Hey2 [98]. These two groups of cells correspond to hair cells and supporting cells. The remaining two clusters of cells are either unlabeled or express EGFP. These cells likely correspond to other nonsensory cells from the medial and lateral regions flanking the sensory epithelium [98]. Additional analysis of 52 EGFPexpressing cells clustered into two main groups of supporting cells that express distinct gene sets. These groups correspond to supporting cells found in the medial and lateral portion of the cochlear sensory epithelia [98]. Transcriptome analysis identifies unique subpopulations of cells within the sensory epithelium and reveals the complexity of cell types even within the cochlear sensory epithelium. A diverse population of nonsensory cells are competent for conversion into SGNs [30, 60]. Identifying an expression signature from these non-sensory cells may help define a competent progenitor state. Similar scRNA-seq experiments from mature SGNs will assist in defining the mature cellular state and reveal the molecular basis of heterogeneity that correspond to SGNs with different electrophysiological properties $[6,102,103]$.

To determine the populations in developing otic cell types, the transcriptome of early developing mouse otic vesicle using a highly parallelized qPCR assay has been done. The expression of 92 marker and 4 control genes allows identification of different cell types and subpopulations of cells within the developing otic vesicle. Transgenic reporter mice containing the $\mathrm{Pax} 2^{\mathrm{Cre}+/}$; $\mathrm{Gt}(\mathrm{ROSA} 26)$ Sor $^{\text {tdTomato,mEGFP }}$ genetically mark cells in the developing otic vesicle [104, 105]. Cells in the otic vesicle and delaminating neuroblasts that undergo Cre recombination express a membrane bound EGFP whereas non-otic cells that do not undergo recombination are marked by tdTomato. Microdissection of the inner ear from these animals followed by FACS allowed separation of developing otic cell types from non-otic cell types in the tissue [106]. Lineage and expression analyses from 382 single cells suggest that distinct populations could be assigned to discrete domains in the developing otic vesicle. By applying multivariate cluster, principal component, and network analyses to the data, populations of sensory progenitors could be distinguished from neuroblasts [106]. Within the neuroblast population, principal component analysis identifies distinct cell types that correspond to early and late delaminating neuroblasts that express the constellation of Neurogl, Isll, and NeuroD1 [106]. Additional network analysis reveals a distinct group of transitioning cells between the two neuroblast populations which reflects the progression of different cellular states during neuronal development [106]. By expanding the differentiation trajectory and determining transition from neurosensory precursors all the way to mature SGNs will provide a molecular roadmap for the proper cellular decisions to guide a progenitor cell into a differentiated SGN.

\section{Conclusions}

The advent of single-cell sequencing has provided a tool to determine the cellular state of a single cell. In addition to the static transcriptome state of a cell, temporal information can be extracted using scRNA-seq data obtained from developing inner ears or differentiating stem cell/progenitor cell cultures. The small number of cells that are harvested and the diversity of distinct cell types from the inner ear sensory organs make these preparations particular well-suited for scRNA-seq analysis. Descriptive anatomy of the inner ear suggests at least eight distinct cell types in the cochlear sensory epithelium (Claudius' cells, Hensen's cells, Dieters' cells, outer hair cells, pillar cells, inner hair cell, inner phalangeal cell, and border cells) [107]. Sequencing more cells and increasing sequencing depth may help determine and refine subpopulation of cells, determine distinct cell types in the cochlea, and identify molecular hallmarks for progenitors that are competent to become SGNs. Extraction of temporal information from scRNA-seq can predict lineage relationships and transitions from a neurosensory precursor to a differentiated state during development. These relationships between cells can be validated by in vivo fate mapping. Differentiation of in vitro generated otic cell types is comprised of a heterogeneous population of cells at different cellular states. Pseudotemporal ordering of the transcriptomes will help determine the differentiation trajectory of stem cell-derived SGNs. Trajectory of these cells will be useful in determining decision points for successful SGNs differentiation or to avoid cellular decisions that lead to continued proliferation or improper differentiation. Identifying the differentiation trajectory of non-sensory supporting cells into SGNs by transcription factor-induced direct conversion can also determine the various states that prevent efficient conversion. Comparison of the differentiation trajectory of all these cell types may reveal a common path that leads to SGN differentiation. 
Currently, eight different transcriptomes have been identified from cells of the sensory epithelium [98] and two neuroblasts populations [106]. These transcriptomes are cells derived from early post-natal and embryonic inner ear, respectively. A complete set of single-cell transcriptome data from development, post-natal, and adult inner ears will provide new insight into the developmental trajectory and lineage of otic cells. For regeneration, the set of single-cell transcriptome data will address whether newly regenerated cells undergo the same differentiation trajectory as observed in development and whether the nascent cells are similar to mature cell types. Most inner ear regeneration studies have been done using early post-natal or juvenile animals. The plasticity and competency of cells from mature animals may be drastically different in adult animals. These differences may be reflected in the transcriptomes.

In addition to the differences in the transcriptomes, epigenetic changes may affect the competency of a cell and create a barrier for regeneration in the adult inner ear. Identifying potential differences in the epigenetic status by interrogating histone marks at a single-cell level will be useful in conjunction with determining the cellular state [108]. Single-cell epigenetic information will help determine the cell types that are amenable for regeneration. Use of scRNA-seq and related single-cell techniques for molecular interrogation provides invaluable information about the cellular states and differentiation trajectory. Using this information, altering the transcriptome or epigenetic state of stem cells, progenitor cells, or non-sensory cells may be used to promote efficient SGN regeneration in the inner ear.

\begin{abstract}
Acknowledgments I thank Susan Xue, Wenke Liu, and Dr. Robin Davis for advice on organotypic cochlear cultures. I would also like to acknowledge Zhichao Song for testing and performing Psd95 antibody immunofluorescence on cochlear explant cultures. The work was supported in part by the Duncan and Nancy MacMillan Faculty Development Chair Endowment Fund, Busch Biomedical Research Grant, Rutgers Faculty Development Grant, and NIDCD R01 DC015000.
\end{abstract}

\section{Compliance with Ethical Standards}

Conflict of Interest The author declares that there is no conflict of interest.

Human and Animal Rights and Informed Consent Mice were kept in the Nelson Labs Animal Facility and used in accordance with the animal protocols.

\section{References}

1. Hudspeth AJ. Integrating the active process of hair cells with cochlear function. Nat Rev Neurosci. 2014;15(9):600-14. doi:10.1038/nrn3786.
2. Fettiplace R, Hackney CM. The sensory and motor roles of auditory hair cells. Nat Rev Neurosci. 2006;7(1):19-29. doi:10.1038 /nrn1828.

3. He DZ, Jia S, Dallos P. Mechanoelectrical transduction of adult outer hair cells studied in a gerbil hemicochlea. Nature. 2004;429(6993):766-70. doi:10.1038/nature02591.

4. Barclay M, Ryan AF, Housley GD. Type I vs type II spiral ganglion neurons exhibit differential survival and neuritogenesis during cochlear development. Neural Dev. 2011;6:33. doi:10.1186 /1749-8104-6-33.

5. Huang LC, Barclay M, Lee K, Peter S, Housley GD, Thorne PR, et al. Synaptic profiles during neurite extension, refinement and retraction in the developing cochlea. Neural Dev. 2012;7:38. doi:10.1186/1749-8104-7-38.

6. Meyer AC, Frank T, Khimich D, Hoch G, Riedel D, Chapochnikov NM, et al. Tuning of synapse number, structure and function in the cochlea. Nat Neurosci. 2009;12(4):444-53. doi:10.1038/nn.2293.

7. Hafidi A. Peripherin-like immunoreactivity in type II spiral ganglion cell body and projections. Brain Res. 1998;805(1-2):18190.

8. Liberman MC. Morphological differences among radial afferent fibers in the cat cochlea: an electron-microscopic study of serial sections. Hear Res. 1980;3(1):45-63.

9. Gillespie PG, Muller U. Mechanotransduction by hair cells: models, molecules, and mechanisms. Cell. 2009;139(1):33-44. doi:10.1016/j.cell.2009.09.010.

10. Vollrath MA, Kwan KY, Corey DP. The micromachinery of mechanotransduction in hair cells. Annu Rev Neurosci. 2007;30: 339-65. doi:10.1146/annurev.neuro.29.051605.112917.

11. Kujawa SG, Liberman MC. Acceleration of age-related hearing loss by early noise exposure: evidence of a misspent youth. $\mathrm{J}$ Neurosci: Off J Soc Neurosci. 2006;26(7):2115-23. doi:10.1523 /JNEUROSCI.4985-05.2006.

12. Kujawa SG, Liberman MC. Adding insult to injury: cochlear nerve degeneration after "temporary" noise-induced hearing loss. J Neurosci: Off J Soc Neurosci. 2009;29(45):14077-85. doi:10.1523/JNEUROSCI.2845-09.2009.

13. Kujawa SG, Liberman MC. Synaptopathy in the noise-exposed and aging cochlea: primary neural degeneration in acquired sensorineural hearing loss. Hear Res. 2015;330(Pt B):191-9. doi:10.1016/j.heares.2015.02.009.

14. Lin FR, Niparko JK, Ferrucci L. Hearing loss prevalence in the United States. Arch Intern Med. 2011;171(20):1851-2. doi:10.1001 /archinternmed.2011.506.

15. Manchaiah VK, Zhao F, Danesh AA, Duprey R. The genetic basis of auditory neuropathy spectrum disorder (ANSD). Int J Pediatr Otorhinolaryngol. 2011;75(2):151-8. doi:10.1016/j. ijporl.2010.11.023.

16. Morton CC. Genetics, genomics and gene discovery in the auditory system. Hum Mol Genet. 2002;11(10):1229-40.

17. Atkinson PJ, Huarcaya Najarro E, Sayyid ZN, Cheng AG. Sensory hair cell development and regeneration: similarities and differences. Development. 2015;142(9):1561-71. doi:10.1242 /dev.114926.

18. Wu DK, Kelley MW. Molecular mechanisms of inner ear development. Cold Spring Harb Perspect Biol. 2012;4(8):a008409. doi:10.1101/cshperspect.a008409.

19. Bok J, Chang W, Wu DK. Patterning and morphogenesis of the vertebrate inner ear. Int J Dev Biol. 2007;51(6-7):521-33. doi:10.1387/ijdb.072381jb.

20. Fekete DM, Wu DK. Revisiting cell fate specification in the inner ear. Curr Opin Neurobiol. 2002;12(1):35-42.

21. Carney PR, Silver J. Studies on cell migration and axon guidance in the developing distal auditory system of the mouse. J Comp Neurol. 1983;215(4):359-69. doi:10.1002/cne.902150402. 
22. Farinas I, Jones KR, Tessarollo L, Vigers AJ, Huang E, Kirstein $\mathrm{M}$, et al. Spatial shaping of cochlear innervation by temporally regulated neurotrophin expression. J Neurosci: Off J Soc Neurosci. 2001;21(16):6170-80.

23. Yang T, Kersigo J, Jahan I, Pan N, Fritzsch B. The molecular basis of making spiral ganglion neurons and connecting them to hair cells of the organ of Corti. Hear Res. 2011;278(1-2):21-33. doi:10.1016/j.heares.2011.03.002.

24. Matei V, Pauley S, Kaing S, Rowitch D, Beisel KW, Morris K, et al. Smaller inner ear sensory epithelia in Neurog 1 null mice are related to earlier hair cell cycle exit. Dev Dyn: Off Publ Am Assoc Anatomists. 2005;234(3):633-50. doi:10.1002/dvdy.20551.

25. Ruben RJ. Development of the inner ear of the mouse: a radioautographic study of terminal mitoses. Acta Otolaryngol. 1967; Suppl 220:1-44.

26. Evsen L, Sugahara S, Uchikawa M, Kondoh H, Wu DK. Progression of neurogenesis in the inner ear requires inhibition of Sox 2 transcription by neurogenin 1 and neurod1. J Neurosci: Off J Soc Neurosci. 2013;33(9):3879-90. doi:10.1523 /JNEUROSCI.4030-12.2013.

27. Fritzsch B, Jahan I, Pan N, Kersigo J, Duncan J, Kopecky B. Dissecting the molecular basis of organ of Corti development: where are we now? Hear Res. 2011;276(1-2):16-26. doi:10.1016/j.heares.2011.01.007.

28. Hagstrom SA, Pauer GJ, Reid J, Simpson E, Crowe S, Maumenee $\mathrm{IH}$, et al. SOX2 mutation causes anophthalmia, hearing loss, and brain anomalies. Am J Med Genet A. 2005;138A(2):95-8. doi:10.1002/ajmg.a.30803.

29. Fantes J, Ragge NK, Lynch SA, McGill NI, Collin JR, HowardPeebles PN, et al. Mutations in SOX2 cause anophthalmia. Nat Genet. 2003;33(4):461-3. doi:10.1038/ng1120.

30. Puligilla C, Dabdoub A, Brenowitz SD, Kelley MW. Sox 2 induces neuronal formation in the developing mammalian cochlea. $\mathrm{J}$ Neurosci: Off J Soc Neurosci. 2010;30(2):714-22. doi:10.1523 /JNEUROSCI.3852-09.2010.

31. Kiernan AE, Pelling AL, Leung KK, Tang AS, Bell DM, Tease C, et al. Sox 2 is required for sensory organ development in the mammalian inner ear. Nature. 2005;434(7036):1031-5. doi:10.1038 /nature 03487.

32. Ma Q, Anderson DJ, Fritzsch B. Neurogenin 1 null mutant ears develop fewer, morphologically normal hair cells in smaller sensory epithelia devoid of innervation. J Assoc Res Otolaryngol: JARO. 2000;1(2):129-43.

33. Liu M, Pereira FA, Price SD, Chu MJ, Shope C, Himes D, et al. Essential role of BETA2/NeuroD1 in development of the vestibular and auditory systems. Genes Dev. 2000;14(22):2839-54.

34. Appler JM, Goodrich LV. Connecting the ear to the brain: molecular mechanisms of auditory circuit assembly. Prog Neurobiol. 2011;93(4):488-508. doi:10.1016/j.pneurobio.2011.01.004.

35. Fritzsch B. Development of inner ear afferent connections: forming primary neurons and connecting them to the developing sensory epithelia. Brain Res Bull. 2003;60(5-6):423-33.

36. Koundakjian EJ, Appler JL, Goodrich LV. Auditory neurons make stereotyped wiring decisions before maturation of their targets. $\mathrm{J}$ Neurosci: Off J Soc Neurosci. 2007;27(51):14078-88. doi:10.1523/JNEUROSCI.3765-07.2007.

37. Moser T, Predoehl F, Starr A. Review of hair cell synapse defects in sensorineural hearing impairment. Otol Neurotology : Off Publ Am Otological Soc, Am Neurotology Soc Eur Acad Otol Neurotology. 2013;34(6):995-1004. doi:10.1097/MAO.0b013 e3182814d4a.

38. Safieddine S, El-Amraoui A, Petit C. The auditory hair cell ribbon synapse: from assembly to function. Annu Rev Neurosci. 2012;35:509-28. doi:10.1146/annurev-neuro-061010-113705.
39. Glowatzki E, Grant L, Fuchs P. Hair cell afferent synapses. Curr Opin Neurobiol. 2008;18(4):389-95. doi:10.1016/j. conb.2008.09.006.

40. Frank T, Rutherford MA, Strenzke N, Neef A, Pangrsic T, Khimich D, et al. Bassoon and the synaptic ribbon organize $\mathrm{Ca}(2)+$ channels and vesicles to add release sites and promote refilling. Neuron. 2010;68(4):724-38. doi:10.1016/j. neuron.2010.10.027.

41. Goutman JD, Glowatzki E. Time course and calcium dependence of transmitter release at a single ribbon synapse. Proc Natl Acad Sci U S A. 2007;104(41):16341-6. doi:10.1073 /pnas.0705756104.

42. Griesinger CB, Richards CD, Ashmore JF. Fast vesicle replenishment allows indefatigable signalling at the first auditory synapse. Nature. 2005;435(7039):212-5. doi:10.1038/nature03567.

43. Matsubara A, Laake JH, Davanger S, Usami S, Ottersen OP. Organization of AMPA receptor subunits at a glutamate synapse: a quantitative immunogold analysis of hair cell synapses in the rat organ of Corti. J Neurosci: Off J Soc Neurosci. 1996;16(14): 4457-67.

44. Yu WM, Appler JM, Kim YH, Nishitani AM, Holt JR, Goodrich LV. A Gata3-Mafb transcriptional network directs post-synaptic differentiation in synapses specialized for hearing. eLife. 2013;2: e01341. doi:10.7554/eLife.01341.

45. Van Esch H, Groenen P, Nesbit MA, Schuffenhauer S, Lichtner P, Vanderlinden G, et al. GATA3 haplo-insufficiency causes human HDR syndrome. Nature. 2000;406(6794):419-22. doi:10.1038 135019088 .

46. Lawoko-Kerali G, Rivolta MN, Lawlor P, Cacciabue-Rivolta DI, Langton-Hewer C, van Doorninck JH, et al. GATA3 and NeuroD distinguish auditory and vestibular neurons during development of the mammalian inner ear. Mech Dev. 2004;121(3):287-99. doi:10.1016/j.mod.2003.12.006.

47. Lawoko-Kerali G, Rivolta MN, Holley M. Expression of the transcription factors GATA3 and Pax2 during development of the mammalian inner ear. J Comp Neurol. 2002;442(4):378-91. doi:10.1002/cne.10088.

48. Appler JM, Lu CC, Druckenbrod NR, Yu WM, Koundakjian EJ, Goodrich LV. Gata3 is a critical regulator of cochlear wiring. J Neurosci: Off J Soc Neurosci. 2013;33(8):3679-91. doi:10.1523 /JNEUROSCI.4703-12.2013.

49. Duncan JS, Lim KC, Engel JD, Fritzsch B. Limited inner ear morphogenesis and neurosensory development are possible in the absence of GATA3. Int J Dev Biol. 2011;55(3):297-303. doi:10.1387/ijdb.103178jd.

50. Luo XJ, Deng M, Xie X, Huang L, Wang H, Jiang L, et al. GATA3 controls the specification of prosensory domain and neuronal survival in the mouse cochlea. Hum Mol Genet. 2013;22(18):360923. doi: $10.1093 / \mathrm{hmg} / \mathrm{ddt} 212$.

51. Duncan JS, Fritzsch B. Continued expression of GATA3 is necessary for cochlear neurosensory development. PLoS One. 2013;8(4):e62046. doi:10.1371/journal.pone.0062046.

52. Yang Y, Cvekl A. Large Maf transcription factors: Cousins of AP1 proteins and important regulators of cellular differentiation. Einstein J Biol Med. 2007;23:2-11.

53. Marrs GS, Spirou GA. Embryonic assembly of auditory circuits: spiral ganglion and brainstem. J Physiol. 2012;590(Pt 10):2391408. doi:10.1113/jphysiol.2011.226886.

54. Liberman MC. Auditory-nerve response from cats raised in a lownoise chamber. J Acoust Soc Am. 1978;63(2):442-55.

55. Martinez-Monedero R, Oshima K, Heller S, Edge AS. The potential role of endogenous stem cells in regeneration of the inner ear. Hear Res. 2007;227(1-2):48-52. doi:10.1016/j. heares.2006.12.015. 
56. Jeon CJ, Strettoi E, Masland RH. The major cell populations of the mouse retina. J Neurosci: Off J Soc Neurosci. 1998;18(21):893646.

57. Oshima K, Grimm CM, Corrales CE, Senn P, Martinez Monedero $\mathrm{R}$, Geleoc GS, et al. Differential distribution of stem cells in the auditory and vestibular organs of the inner ear. J Assoc Res Otolaryngol: JARO. 2007;8(1):18-31. doi:10.1007/s10162-0060058-3.

58. Koehler KR, Mikosz AM, Molosh AI, Patel D, Hashino E. Generation of inner ear sensory epithelia from pluripotent stem cells in 3D culture. Nature. 2013;500(7461):217-21. doi:10.1038 /nature12298.

59. Martinez-Monedero R, Yi E, Oshima K, Glowatzki E, Edge AS. Differentiation of inner ear stem cells to functional sensory neurons. Dev Neurobiol. 2008;68(5):669-84. doi:10.1002 /dneu.20616.

60. Nishimura K, Weichert RM, Liu W, Davis RL, Dabdoub A. Generation of induced neurons by direct reprogramming in the mammalian cochlea. Neuroscience. 2014;275:125-35. doi:10.1016/j.neuroscience.2014.05.067.

61. Fu Y, Ding D, Wei L, Jiang H, Salvi R. Ouabain-induced apoptosis in cochlear hair cells and spiral ganglion neurons in vitro. BioMed Res Int. 2013;2013:628064. doi:10.1155/2013/628064.

62. Lang H, Schulte BA, Schmiedt RA. Ouabain induces apoptotic cell death in type I spiral ganglion neurons, but not type II neurons. J Assoc Res Otolaryngol: JARO. 2005;6(1):63-74. doi:10.1007 /s10162-004-5021-6.

63. Yuan Y, Shi F, Yin Y, Tong M, Lang H, Polley DB, et al. Ouabaininduced cochlear nerve degeneration: synaptic loss and plasticity in a mouse model of auditory neuropathy. J Assoc Res Otolaryngol: JARO. 2014;15(1):31-43. doi:10.1007/s10162013-0419-7.

64. Corrales CE, Pan L, Li H, Liberman MC, Heller S, Edge AS. Engraftment and differentiation of embryonic stem cell-derived neural progenitor cells in the cochlear nerve trunk: growth of processes into the organ of Corti. J Neurobiol. 2006;66(13):1489500. doi:10.1002/neu.20310.

65. Chen W, Jongkamonwiwat N, Abbas L, Eshtan SJ, Johnson SL, Kuhn S, et al. Restoration of auditory evoked responses by human ES-cell-derived otic progenitors. Nature. 2012;490(7419):27882. doi:10.1038/nature11415.

66. Nayagam BA, Edge AS, Needham K, Hyakumura T, Leung J, Nayagam DA, et al. An in vitro model of developmental synaptogenesis using cocultures of human neural progenitors and cochlear explants. Stem Cells Dev. 2013;22(6):901-12. doi:10.1089 /scd.2012.0082.

67. Martinez-Monedero R, Corrales CE, Cuajungco MP, Heller S, Edge AS. Reinnervation of hair cells by auditory neurons after selective removal of spiral ganglion neurons. J Neurobiol. 2006;66(4):319-31. doi:10.1002/neu.20232.

68. Kwan KY, Shen J, Corey DP. C-MYC transcriptionally amplifies SOX2 target genes to regulate self-renewal in multipotent otic progenitor cells. Stem cell Reports. 2015;4(1):47-60. doi:10.1016/j.stemcr.2014.11.001.

69. Spoendlin H, Schrott A. Analysis of the human auditory nerve. Hear Res. 1989;43(1):25-38.

70. Kiang NY, Rho JM, Northrop CC, Liberman MC, Ryugo DK. Hair-cell innervation by spiral ganglion cells in adult cats. Science. 1982;217(4555):175-7.

71. Nayagam BA, Muniak MA, Ryugo DK. The spiral ganglion: connecting the peripheral and central auditory systems. Hear Res. 2011;278(1-2):2-20. doi:10.1016/j.heares.2011.04.003.

72. Hasson T, Gillespie PG, Garcia JA, MacDonald RB, Zhao Y, Yee $\mathrm{AG}$, et al. Unconventional myosins in inner-ear sensory epithelia. J Cell Biol. 1997;137(6):1287-307.
73. Iwafuchi-Doi M, Zaret KS. Pioneer transcription factors in cell reprogramming. Genes Dev. 2014;28(24):2679-92. doi:10.1101 /gad.253443.114.

74. Wapinski OL, Vierbuchen T, Qu K, Lee QY, Chanda S, Fuentes $\mathrm{DR}$, et al. Hierarchical mechanisms for direct reprogramming of fibroblasts to neurons. Cell. 2013;155(3):621-35. doi:10.1016/j. cell.2013.09.028.

75. Nishimura K, Nakagawa T, Sakamoto T, Ito J. Fates of murine pluripotent stem cell-derived neural progenitors following transplantation into mouse cochleae. Cell Transplant. 2012;21(4):76371. doi:10.3727/096368911X623907.

76. Liu XP, Koehler KR, Mikosz AM, Hashino E, Holt JR. Functional development of mechanosensitive hair cells in stem cell-derived organoids parallels native vestibular hair cells. Nat Commun. 2016;7:11508. doi:10.1038/ncomms 11508 .

77. Klein AM, Mazutis L, Akartuna I, Tallapragada N, Veres A, Li V, et al. Droplet barcoding for single-cell transcriptomics applied to embryonic stem cells. Cell. 2015;161(5):1187-201. doi:10.1016/j. cell.2015.04.044.

78. Macosko EZ, Basu A, Satija R, Nemesh J, Shekhar K, Goldman $\mathrm{M}$, et al. Highly parallel genome-wide expression profiling of individual cells using nanoliter droplets. Cell. 2015;161(5): 1202-14. doi:10.1016/j.cell.2015.05.002.

79. Tang F, Barbacioru C, Nordman E, Li B, Xu N, Bashkirov VI, et al. RNA-Seq analysis to capture the transcriptome landscape of a single cell. Nat Protoc. 2010;5(3):516-35. doi:10.1038 /nprot.2009.236.

80. Islam S, Kjallquist U, Moliner A, Zajac P, Fan JB, Lonnerberg P, et al. Characterization of the single-cell transcriptional landscape by highly multiplex RNA-seq. Genome Res. 2011;21(7):1160-7. doi:10.1101/gr.110882.110.

81. Islam S, Zeisel A, Joost S, La Manno G, Zajac P, Kasper M, et al. Quantitative single-cell RNA-seq with unique molecular identifiers. Nat Methods. 2014;11(2):163-6. doi:10.1038/nmeth.2772.

82. Yan L, Yang M, Guo H, Yang L, Wu J, Li R, et al. Single-cell RNA-Seq profiling of human preimplantation embryos and embryonic stem cells. Nat Struct Mol Biol. 2013;20(9):1131-9. doi:10.1038/nsmb.2660.

83. Ilicic T, Kim JK, Kolodziejczyk AA, Bagger FO, McCarthy DJ, Marioni JC, et al. Classification of low quality cells from singlecell RNA-seq data. Genome Biol. 2016;17:29. doi:10.1186 /s13059-016-0888-1.

84. Wu AR, Neff NF, Kalisky T, Dalerba P, Treutlein B, Rothenberg ME, et al. Quantitative assessment of single-cell RNA-sequencing methods. Nat Methods. 2014;11(1):41-6. doi:10.1038 /nmeth.2694.

85. Gawad C, Koh W, Quake SR. Single-cell genome sequencing: current state of the science. Nat Rev Genet. 2016;17(3):175-88. doi:10.1038/nrg.2015.16.

86. Kolodziejczyk AA, Kim JK, Svensson V, Marioni JC, Teichmann SA. The technology and biology of single-cell RNA sequencing. Mol Cell. 2015;58(4):610-20. doi:10.1016/j.molcel.2015.04.005.

87. Vierbuchen T, Wernig M. Direct lineage conversions: unnatural but useful? Nat Biotechnol. 2011;29(10):892-907. doi:10.1038 /nbt.1946.

88. Pollen AA, Nowakowski TJ, Shuga J, Wang X, Leyrat AA, Lui $\mathrm{JH}$, et al. Low-coverage single-cell mRNA sequencing reveals cellular heterogeneity and activated signaling pathways in developing cerebral cortex. Nat Biotechnol. 2014;32(10):1053-8. doi:10.1038/nbt.2967.

89. Sanes JR, Zipursky SL. Design principles of insect and vertebrate visual systems. Neuron. 2010;66(1):15-36. doi:10.1016/j. neuron.2010.01.018.

90. Masland RH. The neuronal organization of the retina. Neuron. 2012;76(2):266-80. doi:10.1016/j.neuron.2012.10.002. 
91. Magwene PM, Lizardi P, Kim J. Reconstructing the temporal ordering of biological samples using microarray data. Bioinformatics. 2003;19(7):842-50.

92. Trapnell C, Cacchiarelli D, Grimsby J, Pokharel P, Li S, Morse M, et al. The dynamics and regulators of cell fate decisions are revealed by pseudotemporal ordering of single cells. Nat Biotechnol. 2014;32(4):381-6. doi:10.1038/nbt.2859.

93. Julia M, Telenti A, Rausell A. Sincell: an R/Bioconductor package for statistical assessment of cell-state hierarchies from single-cell RNA-seq. Bioinformatics. 2015;31(20):3380-2. doi:10.1093 /bioinformatics/btv368.

94. Llorens-Bobadilla E, Zhao S, Baser A, Saiz-Castro G, Zwadlo K, Martin-Villalba A. Single-cell transcriptomics reveals a population of dormant neural stem cells that become activated upon brain injury. Cell Stem Cell. 2015;17(3):329-40. doi:10.1016/j. stem.2015.07.002.

95. Shin J, Berg DA, Zhu Y, Shin JY, Song J, Bonaguidi MA, et al. Single-cell RNA-seq with waterfall reveals molecular cascades underlying adult neurogenesis. Cell Stem Cell. 2015;17(3):360 72. doi:10.1016/j.stem.2015.07.013.

96. Leng N, Chu LF, Barry C, Li Y, Choi J, Li X, et al. Oscope identifies oscillatory genes in unsynchronized single-cell RNAseq experiments. Nat Methods. 2015;12(10):947-50. doi:10.1038/nmeth.3549.

97. Bendall SC, Davis KL, Amir el AD, Tadmor MD, Simonds EF, Chen TJ, et al. Single-cell trajectory detection uncovers progression and regulatory coordination in human B cell development. Cell. 2014;157(3):714-25. doi:10.1016/j.cell.2014.04.005.

98. Burns JC, Kelly MC, Hoa M, Morell RJ, Kelley MW. Single-cell RNA-Seq resolves cellular complexity in sensory organs from the neonatal inner ear. Nat Commun. 2015;6:8557. doi:10.1038 /ncomms9557.

99. Gong S, Zheng C, Doughty ML, Losos K, Didkovsky N, Schambra UB, et al. A gene expression atlas of the central nervous system based on bacterial artificial chromosomes. Nature. 2003;425(6961):917-25. doi:10.1038/nature02033.
100. Yang H, Gan J, Xie X, Deng M, Feng L, Chen X, et al. Gfi1-Cre knock-in mouse line: a tool for inner ear hair cell-specific gene deletion. Genesis. 2010;48(6):400-6. doi:10.1002/dvg.20632.

101. Madisen L, Zwingman TA, Sunkin SM, Oh SW, Zariwala HA, Gu $\mathrm{H}$, et al. A robust and high-throughput Cre reporting and characterization system for the whole mouse brain. Nat Neurosci. 2010;13(1):133-40. doi:10.1038/nn.2467.

102. Liberman LD, Wang H, Liberman MC. Opposing gradients of ribbon size and AMPA receptor expression underlie sensitivity differences among cochlear-nerve/hair-cell synapses. J Neurosci: Off J Soc Neurosci. 2011;31(3):801-8. doi:10.1523 /JNEUROSCI.3389-10.2011.

103. Davis RL, Liu Q. Complex primary afferents: what the distribution of electrophysiologically-relevant phenotypes within the spiral ganglion tells us about peripheral neural coding. Hear Res. 2011;276(1-2):34-43. doi:10.1016/j.heares.2011.01.014.

104. Muzumdar MD, Tasic B, Miyamichi K, Li L, Luo L. A global double-fluorescent Cre reporter mouse. Genesis. 2007;45(9):593605. doi:10.1002/dvg.20335.

105. Ohyama T, Groves AK. Generation of Pax2-Cre mice by modification of a Pax2 bacterial artificial chromosome. Genesis. 2004;38(4):195-9. doi:10.1002/gene.20017.

106. Durruthy-Durruthy R, Gottlieb A, Hartman BH, Waldhaus J, Laske RD, Altman R, et al. Reconstruction of the mouse otocyst and early neuroblast lineage at single-cell resolution. Cell. 2014;157(4):964-78. doi:10.1016/j.cell.2014.03.036.

107. Van De Water TR. Historical aspects of inner ear anatomy and biology that underlie the design of hearing and balance prosthetic devices. Anat Rec (Hoboken). 2012;295(11):1741-59. doi:10.1002/ar.22598.

108. Rotem A, Ram O, Shoresh N, Sperling RA, Goren A, Weitz DA, et al. Single-cell ChIP-seq reveals cell subpopulations defined by chromatin state. Nat Biotechnol. 2015;33(11):1165-72. doi:10.1038/nbt.3383. 